\title{
The Key Components Design and Research of the Projectile System of Marine Pipeline Concrete Weight Coating Production Line
}

\author{
Shuang Jing ${ }^{1,2}$, Zhiqiang Huang ${ }^{1}$, Xu Luo ${ }^{1}$, Kun Zhang ${ }^{1}$ \\ ${ }^{1}$ MOE Key Lab of Oil and Natural Gas Equipment, Southwest Petroleum University, Chengdu, \\ Sichuan, 610500 \\ ${ }^{2}$ Engineering Training Center, Southwest Petroleum University, Chengdu, Sichuan, 610500
}

\begin{abstract}
Keywords: Marine pipe; Concrete weight; Projectile system; Modal analysis
Abstract: Due to the effect of the marine environment, the marine pipelines would drift away because of the water buoyancy when the pipeline laying and operation, as well as suffer the damage from the other external factors. Therefore, we need to coat a concrete layer on the anticorrosive pipe's surface with a certain thickness after the steel cage installed process, namely marine pipeline concrete weight coating (CWC). The concrete coating is the most important section of the marine pipeline CWC process. It's also the key to guarantee the pipe quality. Besides, the projectile device is the significant equipment of pipeline coating, and its performance would directly affect the safety, stability and reliability of coating process, as well as the CWC quality. Through the research of the projectile process at home and abroad, a new vertical projectile method has been proposed. Based on this, we design the concrete projectile system. Therein, the mechanical part of the whole projectile system has been designed. Firstly, the strength and rigidity of the key components of the projectile wheel sets have been checked with the software ANSYS. The check results meet the design requirements. The natural frequency of the projectile bench is determined by the modal analysis. And then we could find out the effect of the motor frequency acting on the resonance damage. According to the above research results, a series of improving methods have been proposed.
\end{abstract}

\section{Introduction}

With the rapid development of China's economic, the requirement of oil and gas resource increase dramatically. Recently, the foreign dependency of our crude oil has been rising continuously, reaching up to $60 \%$. The foreign dependency of crude oil is an important sign to evaluate a country's oil supply safety. The continuously increment of oil requirement indicates that the shortage of oil resource become one of our critical energy problem. As gradual depletion of our inland oil resource, marine oil and gas resource exploration would be drawn more and more attention. The rich marine oil and gas should be made well use of, to relief the increment of resource requirement. Therefore, a series of marine oil and gas exploration projects have been started, and a large member of submarine pipelines would be constructed [1-3]. Due to the effect of the marine environment, the marine pipelines would drift away because of the water buoyancy when the pipeline laying and operation, as well as suffer the damage from the other external factors. The marine pipeline concrete weight coating $(\mathrm{CWC})$ is an effective method to prevent pipelines from being drifted away, as well as the concrete coating has great mechanical damage resistance performance, which could be adapted well to the harsh marine environment and suffer from the external damage. Then the marine s could be protected greatly [4-5].

The marine pipeline CWC is to lay concrete coating (a certain thickness) on the anticorrosive pipeline surface, to increase the whole density of pipe body, thereby overcoming its buoyancy and improving its mechanical protection [6]. The basic processes of CWC include the anticorrosive pipeline production, pipe weighting, online spark testing, stiffener installation, concrete coating and concrete curing. Among them, the concrete coating is the critical process to guarantee CWC quality.

Through the investigation research, our CWC technology is not mature, without our homemade designed CWC devices. In foreign countries, concrete coating processes are mainly divided into two methods, the projectile and intertwine type. Therein, the intertwine type method is very high in cost, low efficient and its coating thickness would be never over $80 \mathrm{~mm}$. Therefore, it is narrowly 
used and not suitable for thick concrete coating, it could not meet our marine pipeline weight coating requirements. The horizontal projectile method is widely used in foreign country. Once the pipe diameter changes continuously, two projectile wheels position should be adjusted accordingly. It is difficult to match the projectile port to the pipe centerline. At the same time, the concrete would drop by a little distance before coated on the pipe surface because of gravity, reducing the concrete adhesion and coating efficiency [7-10]. Therefore, according to the requirements of CWC at home, such as concrete coating thickness, pipeline diameter and cost, we design a new vertical projectile method as the concrete coating process. In this paper, aimed for the new designed vertical projectile method, we have designed the whole projectile system of CWC, which include its mechanical part and hydraulic control. In order to make the designed projectile wheel set reliable, the strength and stiffness of the projectile shaft have been checked. To avoid the resonance damage of the projectile bench, we perform the modal analysis and safety evaluation of projectile bench. The successful design of projectile system has a great significant to our marine pipeline's development.

\section{The structure and design requirement of the projectile system}

The function of the projectile system is to complete the concrete coating process of marine pipelines. In the process of the marine pipeline $\mathrm{CWC}$, the anticorrosive pipe with steel cage installed is loaded onto a coating cart, and then the pipe is ready for concrete coating process, namely concrete projectile system. The main function of the projectile system should be composed of the concrete coating, concrete amending and plastic film wrapping, according to the requirements of coating process and CWC healthy process.

The composition of projectile system: According to the designed vertical projectile method and its functions, the projectile system structure is shown in Fig. 1.

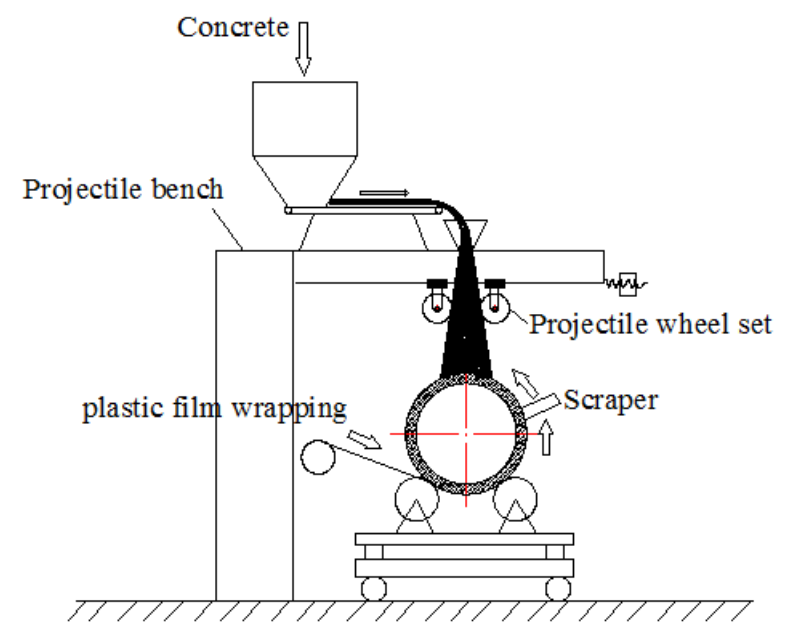

Fig. 1 The schematic diagram of projectile system

It is shown as in Figure.1. The composition of the projectile system should include projectile wheels, projectile bench, scraper apparatus and plastic film wrapping device. The projectile wheel set is the key component of CWC. The concrete would be transported into the gap between the high rotation speed projectile wheels by the feeding belt. The deployed concrete would be high-speed sprayed onto the anticorrosive pipe surface, which is well installed steel cage, thereby achieving the marine pipe's concrete coating process. The rotational speed of projectile wheel set is controlled by the inverter motor, and the interval distance of projectile wheel set is adjusted by the hydraulic system. The projectile bench is another important component. The entire projectile wheel sets are placed on the projectile bench, which withstand the overall weight of the projectile wheels and shock from high speed rotation. After concrete coating, the scraper would be used to trim the uneven coating layer, making it look smooth to meet the $\mathrm{CWC}$ technical requirements. The plastic film could be twined when the pipe is spiral forward at the same time of CWC. That is the plastic film twining process and is good for later healthy keep.

The design requirement of projectile system: (1) The basic design parameters of projectile 
system. Pipe diameter: $\Phi 150-\Phi 1200 \mathrm{~mm}$; Pipe length: 6-12m; The maximum pipe wall thickness: $30 \mathrm{~mm}$; Projectile wheel diameter: $\Phi 598 \mathrm{~mm}$; Rotation speed of projectile wheel: $20-60 \mathrm{~m} / \mathrm{s}$; Length of projectile wheel: $800 / 1200 \mathrm{~mm}$; Thickness of concrete layer: $40-120 \mathrm{~mm}$; The maximum density of concrete: $\geq 3040 \mathrm{~kg} / \mathrm{m}^{3}$; The concrete adhesion rate: $\geq 60 \%$. (2)The design requirement. Due to the impact of local water depth, the marine environment and transportation amount, the pipeline's diameter, length and the counterweight thickness could be changed. In order to meet the performance requirements of $\mathrm{CWC}$, the projectile wheel should be designed according to the following principles: 1) The length of the projectile wheel sets can be adjusted freely to adapt to the concrete coating under the different length, diameters and coating thickness of anticorrosive pipes; 2) The rotation speed of projectile wheel sets should be adjustable; 3) The space of projectile wheel sets should be adjustable; 4) the projectile wheel sets should be easy in structure, stable and reliable, convenient to operate, and easy to maintenance.

\section{The design of key component for projectile system}

The projectile wheel set and bench are the key components of the projectile system. The former is critical part to realize the concrete coating. All the projectile wheel sets are placed on the projectile bench, whose properties directly affect whether the wheel sets could achieve the coating function safely. Therefore, the successful design of projectile wheel sets and bench are the significant of the projectile system.

The design of projectile wheel set. First, we perform the design of projectile wheel sets. As it is discussed before, the concrete is sprayed onto the surface of anticorrosive pipe by the high speed rotation projectile wheel sets. Then pipe could be successful coated to meet technology requirements. Therein, its projectile shaft is the key part, whose strength and stiffness would have effect on the projectile wheel set safety operation.

The basic parameters of projectile wheel set. According to the actual production requirements of concrete coating, the basic design parameters of the whole projectile system, the basic design parameters of projectile wheel sets are determined as follows.

The maximum concrete requirements: $100 \mathrm{~m}^{3} / \mathrm{h}$; The inner diameter of projectile wheel sheet: $\Phi 110 \mathrm{~mm}$; The outer diameter of projectile wheel sheet: $\Phi 598 \mathrm{~mm}$; The length of projectile wheel set: $800 / 1200 \mathrm{~mm}$; The tangential velocity of projectile wheel sheet: $20-60 \mathrm{~m} / \mathrm{s}$; The motor power: $45 \mathrm{~kW}$; The interval of two projectile wheels: $5-300 \mathrm{~mm}$; The rotation speed of motor (matched withФ598 projectile wheel): $639-1917 \mathrm{r} / \mathrm{min}$.

The overall design of projectile wheel set. The projectile wheel sets have been placed on the projectile bench, it is called the upper install method. The projectile wheels are driven by the motor, whose rotation speed is controlled by the inverter. Then the high rotation speed projectile wheel could spray the concrete onto the pipe surface, realizing the concrete coating. The sliding distance of the projectile wheel sets could be controlled by the length of the telescopic cylinder. The projectile wheel set structure is shown as in Fig. 2. It is consist of motor, projectile wheel, projectile shaft, hydraulic components and projectile baseplate.

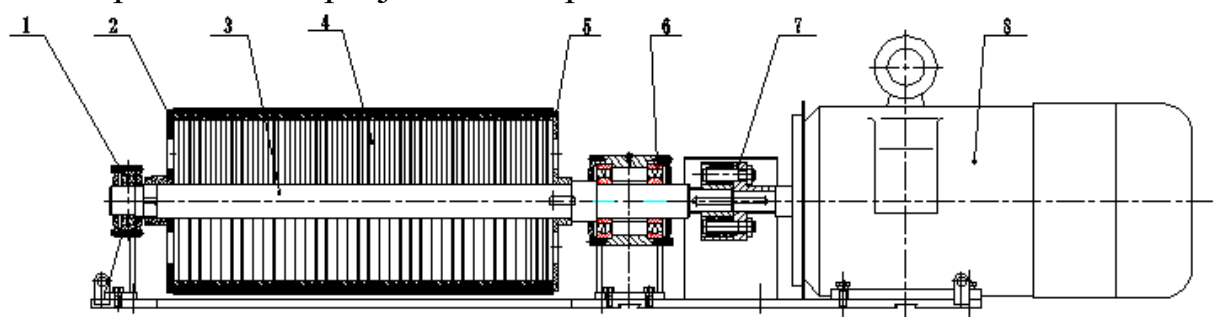

1-Bearing; 2- Platen; 3-Projectile shaft; 4- Projectile wheel;

5- Drive disk; 6-Bearing; 7- Coupling; 8-Motor

Fig. 2 The design drawing of projectile wheel

The wheel sets are superimposed of several projectile wheel sheets, whose outer diameter is $598 \mathrm{~mm}$, inner diameter is $110 \mathrm{~mm}$ and thickness is $34 \mathrm{~mm}$.

(1) The function of controllable speed. According to the basic parameters of concrete projectile 
system, $45 \mathrm{kw}$ inverter motor is used to drive the projectile wheel rotate. Therein, two sets of projectile wheels are controlled respectively by two motors. In working condition, the motion is transported from motor to the projectile shaft, then to the drive disk and projectile wheels. No keyways is designed on the platen, we therefore use the joint action of locking nut and sleeve to compact the platen and projectile wheel sheet without any slide, thereby realizing the normal rotation and frequency control of projectile wheel.

(2) The function of controllable interval. The function of controllable interval is realized by the hydraulic cylinders and T-slot combination scheme. The two sets of projectile wheels are placed on two projectile plates respectively, which are rectangular plates with holes. At the bottom of the projectile plates, the lugs is set to lift the wheel sets, it is easy to install. Each projectile wheel set is driven by two hydraulic cylinders, the projectile wheel sets are driven to slide on the platform board by the hydraulic cylinder telescopic, enabling the space adjustment between the two wheels. After the interval is in suitable position, the projectile wheels would be locked to ensure that the two projectile wheels connection line center is always aligned with the pipe axis.

The design of projectile shaft. The projectile shaft is the key component of projectile wheel set. Its performance affects directly the safety of projectile wheel set. Therefore, we focus on the design and mechanic analysis of projectile shaft.

(1) The design requirement of projectile shaft. The design of projectile shaft should be met the following requirements: 1) The size matching requirement should be satisfied. The size of projectile shaft should get well match with that of projectile wheel. According to the inner diameter of projectile sheet, the shaft diameter of projectile shaft (suffered from the load) is designed to be $110 \mathrm{~mm}$. Because the maximum total length of projectile wheel sets after assembled should be $1224 \mathrm{~mm}$, the main load suffered section length should be designed to meet the maximum total length requirement. 2) The strength requirement should be satisfied. As the projectile shaft is the key component of projectile wheel set, which is subjected to the bending moment and torque. We therefore need to make the mechanic analysis of shaft, thereby meeting its strength requirement. 3) The stiffness requirement should be satisfied. The stiffness requirement is mainly the torque resistance and bending resistance ability standard, which are respectively indicated by the corner and the deflection. In the design process, the corner and the deflection should not be overlarge. 4) The requirement of critical rotation speed. The working rotation speed of projectile shaft should not be close to the critical rotation speed (namely the natural frequency), to avoid the resonance.

(2) The size design of projectile shaft. According to the design requirement, the material of projectile shaft is chosen to be $40 \mathrm{Cr}$, it is very high in strength limit. The shaft structure and size are shown in Fig. 3.

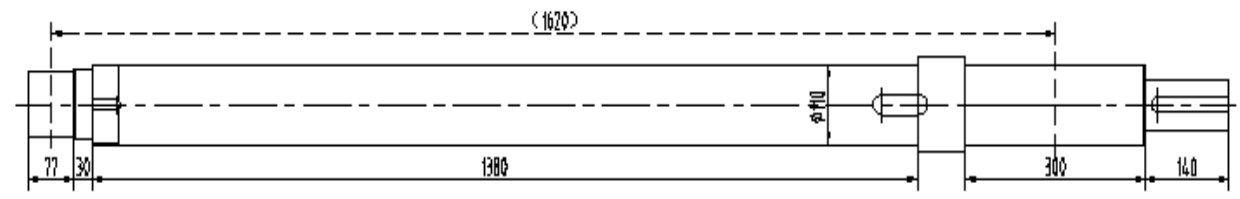

Fig. 3 The projectile shaft

The key coupling shaft sections between the projectile shaft and wheels are designed according to their size matching requirements. The size and structure of the other shaft section are designed by experience and key shaft section size requirement. The key component of projectile wheel sets is the shaft, which is suffered from the bending moment and torque. The strength and stiffness standard are strictly required. We therefore should make the mechanical analysis of shaft to meet the strength requirements.

(3) Mechanical Analysis. According to the actual situation, the simplified simulation model of the projectile shaft is built up. We use the 3D modeling software to build its geometric model, and develop the mechanical analysis after importing it to the FEA software. The results are as are shown in Fig. 4. 


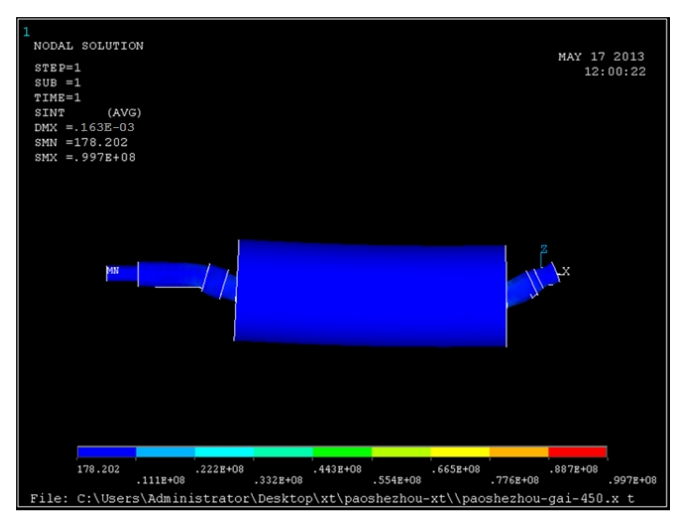

(a) Force distribution

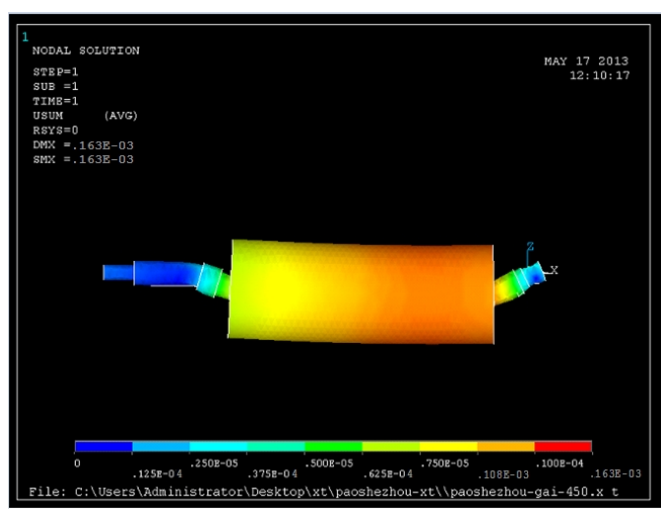

(b) Deformation

Fig. 4 The force and deformation results analysis of shaft

The analysis results are shown as follow: 1) The maximum stress of shaft is $99.7 \mathrm{MPa}$, the yield strength of $40 \mathrm{Cr}$ is $500 \mathrm{Mpa}$, its allowable fatigue stress is $177-213 \mathrm{MPa}$, so the shaft meets the strength requirement. 2) The maximum bending deformation is $0.163 \mathrm{~mm}$, its allowable bending deformation $[\mathrm{y}]$ is defined as the 0.0002 times the distance between bearings, namely $[\mathrm{y}]=0.0002$ $\mathrm{L}=0.324 \mathrm{~mm}$, the allowable bending deformation is larger than maximum bending deformation. As a result, both the strength and stiffness of projectile shaft meet the requirements.

(4) The analysis of critical rotation speed. When the projectile shaft is subject to the outer vibration, once the outer vibration frequency is equal to the natural frequency of projectile shaft, the operation would be unstable and serious vibration, it is called resonance. At this time, the rotation speed of projectile is the critical speed. If the rotation speed of projectile shaft remains the same as the critical speed for a certain time, the shaft deforms more and more seriously, which would have negative effect on its safety operation. The projectile shaft is in high rotation speed, reach up to 1917r/min. Therefore, we need to calculate the critical speed, to make the working rotation speed (n) of shaft away from the critical speed, thereby avoiding the resonance. The first five modals of projectile shaft have been calculated by ANSYS modal analysis, the critical speed of each modal are obtained. It is shown in Table.1.

\begin{tabular}{ccc}
\multicolumn{3}{c}{ Table 1 The natural frequency and critical rotation speed of each order } \\
\hline Order & $\begin{array}{c}\text { Natural frequency } \\
(\mathrm{HZ})\end{array}$ & $\begin{array}{c}\text { Critical rotation speed (r/min) } \\
1\end{array}$ \\
\hline 104.52 & 6271 \\
2 & 373.95 & 22437 \\
3 & 374.69 & 22481 \\
4 & 599.98 & 35999 \\
5 & 733.76 & 44026 \\
\hline
\end{tabular}

From the Table 1, the working rotation speed of projectile shaft is much slower than the first modal critical speed. As this shaft is rigid shaft, so $\mathrm{n}<(0.75-0.8) \mathrm{nc} 1$. from the analysis result, the working rotation speed $\mathrm{n}=1917 \mathrm{r} / \mathrm{min}<<(0.75-0.8) \mathrm{nc} 1=(0.75-0.8) \times 6271=4703-5016 \mathrm{r} / \mathrm{min}$. The projectile shaft meets the working requirements and design requirements.

The design of projectile bench. The projectile bench is another important component of projectile device. The entire projectile wheel sets are placed on the projectile bench, which withstand the overall weight of the projectile wheels and shock from high speed rotation. Its performance highly affects the safe and reliable working of projectile process. In the process of projectile wheel set high speed rotation, the bench would suffer the effect of mechanical vibration. In order to guarantee the safety of projectile system, the vibration performance of bench has been analyzed, thereby providing the guidance to reduce the resonance.

The design of projectile bench. The projectile bench is mainly composed of support frame, projectile plate and other accessories. Therein, the projectile support frame is composed of column, 
baseplate and diagonal rib. The support frames are all welding parts, which are welded by square tube steel $(17 \mathrm{~mm}$ in wall thickness, 2800 in height) and steel plate $(500 \times 500 \mathrm{~mm}, 16 \mathrm{~mm}$ in thickness). The baseplate is fixed to the ground by anchor bolts. The projectile plate is composed of platform roof and strengthening ribs. Take the operation condition needs into consideration, the total length of plate is $6340 \mathrm{~mm}$, width is $4000 \mathrm{~mm}$. The platform roof is welded by 5 standard steels. The projectile wheel sets are placed in the center of the platform, where is reinforced by $30 \mathrm{~mm}$ load-bearing steel. The platform surroundings are non-load bearing zones. It is welded by 4 tread plates $(3 \mathrm{~mm}$ in thickness). Below the roof, it is reinforced by welding ribs. Other accessories include inferior hopper, fence and escalators, which are designed according to the designed standard.

The modal analysis of projectile bench. As the maximum speed of projectile wheel set is 60 $\mathrm{m} / \mathrm{s}(1917 \mathrm{r} / \mathrm{min})$. The high speed would bring the periodicity vibration and shock to the platform, which has bad effect to the stability and reliability operation. Therefore, it is necessary to develop the modal analysis of projectile bench, and find its natural frequency, to keep away from the motor frequency. Thereby, we could prevent the platform and motor from resonance, to guarantee the long-term performance and safety.

(1) The evaluation criterion of vibration stability

The vibration modal is the inherent and entire property of the elastic structure. We could make clear each modal characteristic within a certain frequency and predict the actual structural vibration response of this band under all the vibration resource. The regular evaluation criterion of vibration stability is defined as follow: Keep the self-excited oscillation frequency of mechanical component away from that of the vibration resource. It is given by:

$$
f_{p}<0.85 f \text { or } f_{p}>1.15 f
$$

Where $\mathrm{fp}$ is self-excited oscillation frequency; $\mathrm{f}$ is vibration resource frequency.

When $0.85 \mathrm{f} \leq \mathrm{fp} \leq 1.15 \mathrm{f}$, the mechanical component is in unstable state. We therefore can infer the vibration resource frequency range under the unstable state. It is given by

(2) The modal analysis and results

$$
\frac{f_{p}}{1.15} \leq f \leq \frac{f_{p}}{0.85}
$$

The projectile bench structure has been simplified as follows: (a) The threaded holes and T-slot have been hidden; (b) The welded plate is considered as a part of platform, the welds are ignored and the pattern of the diamond plate is deleted. The whole size is unchanged. (c) The couplings, bearding, seat and projectile wheel sheet of projectile wheel set have been simplified, the weight of projectile wheel set is unchanged before and after simplifying. The 3D model (Fig. 5) of projectile wheel set is built up and imported into the FEA software, the mesh and boundary condition are done. The natural frequency of first 6 order modal have been obtained, it is shown as in Table 2.

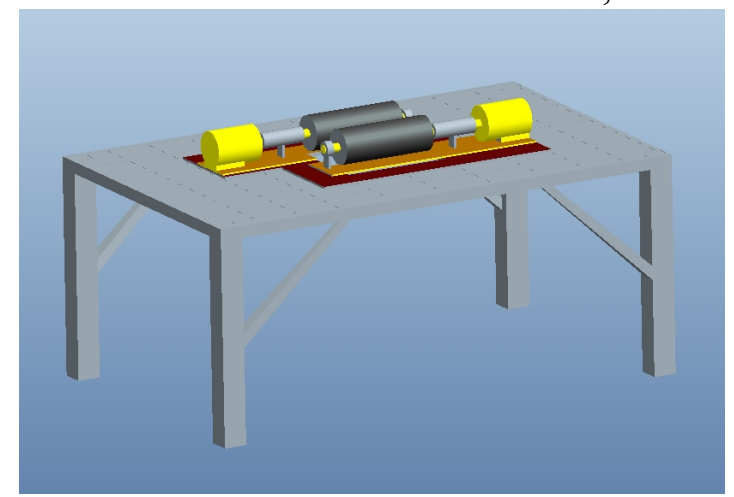

Fig. 5 The model of projectile bench after simplifying 
Table.2 Each modal value of projectile bench and motor frequency

\begin{tabular}{ccccc}
\hline $\begin{array}{c}\text { Orde } \\
\mathrm{r}\end{array}$ & $\begin{array}{c}\text { Natural } \\
\text { frequency }(\mathrm{HZ})\end{array}$ & $\begin{array}{c}\text { Frequency of } \\
\text { unstable excitation } \\
\text { source }(\mathrm{HZ})\end{array}$ & $\begin{array}{c}\text { Maximum } \\
\text { deformation }(\mathrm{mm})\end{array}$ & $\begin{array}{c}\text { Working frequency } \\
\text { scope of motor (HZ) }\end{array}$ \\
\hline 1 & 8.8326 & $7.681-10.391$ & 14.0 & \\
2 & 9.6064 & $8.353-11.302$ & 11.1 & \\
3 & 12.447 & $10.823-14.644$ & 12.4 & $9.053 \sim 31.954$ \\
4 & 17.837 & $15.510-20.985$ & 21.2 & \\
5 & 21.692 & $18.863-25.520$ & 21.0 & \\
6 & 28.208 & $24.529-33.186$ & 27.2 & \\
\hline
\end{tabular}

It is known from the Table.2:

(1) The natural frequency of projectile bench is increasing with order increment, and the frequency increment of two adjacent orders is small, which increases the probability of motor frequency closing to the unstable excitation source frequency, thereby causing large kinds of vibration response.

(2) The first 6 order natural frequencies are all smaller than the maximum motor working frequency, the short-term resonance would happen at the moment of motor start and stop, with temporary vibration and deformation. But the force and deformation is tiny, the design meets the requirement.

(3) Because the natural frequency of bench increases with the order, so if the baseband of bench could be improved to be higher than motor working frequency. The resonance would not occur any more. According to the projectile bench structure, we could appropriately increase the thickness of column and set reinforcing rib plate to improve its natural frequency, reducing the resonance damage.

\section{Conclusions}

Through the investigation of coating process at home and abroad, a new vertical projectile method has been designed. Based on this, the whole mechanical component has been designed. In order to keep the system safe and reliable, its key components (the projectile wheel set and bench) have been designed in detail. The statics and modal of these components have also been analyzed. The conclusions could be drawn as follows:

(1) The projectile shaft has been designed to meet the basic parameters of projectile wheel set, and the static analysis has been finished to verify that the strength and stiffness meet the requirements. Through the modal analysis of projectile shaft, its natural frequency and critical rotation speed are much higher than the motor's working frequency and speed, avoiding the resonance and meeting the working requirements.

(2) The design of projectile bench has been finished. We make the modal analysis to find its natural frequency and maximum deformation. According to the stability evaluation, the resonance of bench might occur within the motor working frequency arrange, but the deformation is tiny. To improve the safety and reliability of projectile bench, we propose a series of improvements about its vibration response characteristics.

\section{Acknowledgments}

This work was financially supported by the major project of China Petroleum Pipeline Bureau, Technology and Equipment research for Submarine Pipeline Corrosion Concrete Coating. 


\section{Reference}

[1] Li Xia. China dependence on foreign oil research. The research of China's dependence on foreign oil. Economic Research Guide, 2011, (31). (In Chinese)

[2] hou Liangliang. The thinking behind China's oil import dependency information. The journal of Zhengzhou Institute of Aeronautical Industry Management, 2012, 30 (5). (In Chinese)

[3] Gong Yanfen, Yang Wenbin, Tan Shudong. Summary of the South China Sea oil and gas resources and the development of strategic vision. Marine Geology and Quaternary Geology, 2012, 32 (5). (In Chinese)

[4] Zhang Xiaoling. Single steel pipe insulation with new products in the offshore oil field. Petroleum Engineering Construction, 2009, 35 (3): 22-26. (In Chinese)

[5] Wang Jinying. The design problem of submarine pipeline concrete weight layer. Chinese offshore oil and gas (Engineering), 1990, 2 (1): 25-28. (In Chinese)

[6] Du Zhongqiang, Li Yukun, et al. Fracture Analysis and Improvement Measures of submarine pipeline concrete weight layer. Petroleum Engineering Construction, 2006, 32 (1): 40-42. (In Chinese)

[7] Offshore Standard DNV-OS-F101. Submarine Pipeline Systems[S]. 2000.

[8] DNV RP E305. On-bottom Stability Design of Submarine Pipelines[S]. 2000.

[9] Li Yukun. The coating equipment and production technology of submarine pipeline concrete weight. Petroleum Engineering Construction, 2005, 31 (5). (In Chinese)

[10] Ding Xinlong, Han Xueyan, et al. Analysis on technique of aggravation of submarine pipeline with concrete. Petroleum Engineering Construction, 2007, 33 (4). (In Chinese) 